\title{
Microbiomes of Muricea californica and $M$. fruticosa: Comparative Analyses of Two Co-occurring Eastern Pacific Octocorals
}

\author{
Johanna B. Holm* and Karla B. Heidelberg \\ Division of Marine Environmental Biology, Department of Biological Science, University of Southern California, Los Angeles, \\ CA, USA
}

Octocorals are sources of novel but understudied microbial diversity. Conversely, scleractinian or reef-building coral microbiomes have been heavily examined in light of the threats of climate change. Muricea californica and Muricea fruticosa are two co-occurring species of gorgonian octocoral abundantly found in the kelp forests of southern California, and thus provide an excellent basis to determine if octocoral

OPEN ACCESS

Edited by:

Christine Moiss/-Eichinger, Medical University Graz, Austria

Reviewed by:

Julie L. Meyer,

University of Florida, USA

Devin Coleman-Derr,

USDA-ARS and University of

California, Berkeley, USA

Erik Cordes,

Temple University, USA

${ }^{*}$ Correspondence:

Johanna B. Holm

jholm@som.umaryland.edu

Specialty section:

This article was submitted to

Microbial Symbioses,

a section of the journal

Frontiers in Microbiology

Received: 21 February 2016

Accepted: 27 May 2016

Published: 21 June 2016

Citation:

Holm JB and Heidelberg KB (2016)

Microbiomes of Muricea californica

and M. fruticosa: Comparative

Analyses of Two Co-occurring Eastern

Pacific Octocorals.

Front. Microbiol. 7:917.

doi: 10.3389/fmicb.2016.00917 microbiomes are host specific. Using Illumina MiSeq amplicon sequencing and replicate samples, we evaluated the microbiomes collected from multiple colonies of both species of Muricea to measure both inter- and intra-colony microbiome variabilities. In addition, microbiomes from overlying sea water and nearby zoanthids (another benthic invertebrate) were also included in the analysis to evaluate whether bacterial taxa specifically associate with octocorals. This is also the first report of microbiomes from these species of Muricea. We show that microbiomes isolated from each sample type are distinct, and specifically, that octocoral species type had the greatest effect on predicting the composition of the Muricea microbiome. Bacterial taxa contributing to compositional differences include distinct strains of Mycoplasma associated with either M. californica or M. fruticosa, an abundance of Spirochaetes observed on M. californica, and a greater diversity of $\gamma$-Proteobacteria associated with $M$. fruticosa. Many of the bacterial taxa contributing to these differences are known for their presence in photosymbiont-containing invertebrate microbiomes.

Keywords: microbiome, octocoral, zoanthid, amplicon sequencing, microbial diversity, MiSeq

\section{INTRODUCTION}

Corals and their microbiomes (including microbial eukaryotes, bacteria, archaea, and viruses) together comprise the entire metaorganism (Bosch and McFall-Ngai, 2011), and these symbiotic associations are critical to host survival (Rosenberg et al., 2007; Bourne and Webster, 2013). Members of these microbiomes contribute to shared metabolic functions, such as nutrient acquisition, environmental sensing, and protection from disease (Rohwer et al., 2002; Reshef et al., 2006; Vega Thurber et al., 2009; Thomas et al., 2010). Despite the perceived importance, clear factors shaping a coral's microbial composition have yet to be fully discerned (Bourne and Webster, 2013). 
Most coral microbiome studies have focused on reef-building scleractinian corals. Few studies have examined the diversity and composition of gorgonian-associated microbial communities (Brück et al., 2007; Webster and Bourne, 2007; Gray et al., 2011; Bourne et al., 2013; Correa et al., 2013) and fewer have examined those of temperate gorgonians (La Rivière et al., 2013; Vezzulli et al., 2013). The gorgonian microbiomes described to date were targeted using culture-based, fingerprinting, and/or clone library analyses [except Bourne et al., 2013, which uses 454 pyrosequencing]. Such strategies are capable of providing taxonomic resolution but capture only a small portion of total microbial diversity. Additionally, there are no studies to our knowledge that statistically compare gorgonian-associated prokaryotic diversity to the surrounding sea water, other benthic organisms, or co-occurring sister species, which would highlight unique niches provided by each host type from the same environment.

The previous studies described above show gorgonianassociated microbial communities from various environments, including the Great Barrier Reef, the deep-sea, and the Mediterranean Sea, were dominated by Gammaproteobacteria (i.e., Endozoicomonas) or Tenericutes (especially Mycoplasma). However, it is intriguing that similar bacterial classes dominate the observed microbiomes of these gorgonian genera located in vastly different (and, in some cases, extreme) marine environments, and raises the question of how much the environment influences microbiome composition. This highlights a need to more deeply examine the gorgonianassociated prokaryotic community with high sampling effort and biological replication, in order to more fully characterize prokaryotic diversity.

We evaluated the microbiomes of two species of Muricea, a genus of azooxanthellate (Van Oppen et al., 2005) gorgonian octocorals found throughout the tropical and temperate eastern Pacific and western Atlantic oceans. Muricea californica and M. fruticosa co-exist in the temperate kelp forests of southern California and are easily distinguished from each other by the colors of their polyps, golden-orange or white, respectively (Grigg, 1970). Their overlapping habitats, similar abundances, colony structures, and general life histories make these species of Muricea suitable for comparison. Multiple colonies of both species were sampled with biological replication to determine the mean microbiome compositions for each colony. To test the specificity of microbial associations, we attempted to maximize intra-colony and inter-colony microbiome variations by purposely sampling colonies from different depths. To further evaluate the presence of gorgonian-specific associations, we also examined the microbiomes of nearby Parazoanthus lucificum (zoanthid, suborder Macrocnemia) colonies in addition to the surrounding sea water. Azooxanthellate P. lucificum (also referred to as Savalia lucifica, Sinniger et al., 2013), named for the brilliant bioluminescence it emits, was specifically chosen as a comparative organism because it occupies similar space as Muricea colonies in both the water column and the benthos due to its life-history trait of infecting and overgrowing M. californica colonies (Cutress and Pequegnat, 1960). To our knowledge, only one other zoanthid (suborder: Brachycnemina) microbiome has been described (Sun et al., 2014). Herein, we characterize and compare deeply sequenced microbiomes of M. californica, M. fruticosa, and P. lucificum, and in addition, examine gorgonian-associated microbes via light and fluorescence microscopy from mucus and polyp tissue to better understand bacterial micro-niches.

\section{MATERIALS AND METHODS}

\section{Sample Collection}

All sample collections were made in accordance with CADFW Scientific Collecting Permit \#12734, issued to J. Holm. $M$. californica $(\mathrm{Mc})$ and $M$. fruticosa (Mf) samples were collected in replicate in addition to nearby samples of the zoanthid, P. lucificum (Pl). Samples were collected from a rocky wall of Santa Catalina Island, CA (33 $\left.26^{\prime} 53.9^{\prime \prime} \mathrm{N}, 118^{\circ} 28^{\prime} 42.3^{\prime \prime} \mathrm{W}\right)$ midday on October 14, 2013. Muricea species were distinguished using morphological characters previously described (Grigg, 1970).

For each Mc and Mf species, subsamples from three colonies from different depths were sampled in situ (range: 8-16 m depth). Collection depth is indicated in the sample's name, following the replicate branch number. Muricea colonies are $<1 \mathrm{~m}$ wide so, the distances between branch replicates from the same colony were no more than $1 \mathrm{~m}$ (i.e., colonies were no more than $1 \mathrm{~m}$ wide). Colonies from similar depths were $>1 \mathrm{~m}$ but no more than 3-4 $\mathrm{m}$ apart. Sampling techniques to reduce contamination were employed; samples were captured in $50 \mathrm{~mL}$ conical tubes without handling. Additionally, single branches from two $\mathrm{Pl}$ colonies from depths 9 and $16 \mathrm{~m}$ growing adjacent to the 11-12 $\mathrm{m}$ gorgonian colonies, were collected. The zoanthid samples were cut and collected in a similar manner as Muricea samples. One liter of SW was collected from $12 \mathrm{~m}$ depth ca. $2 \mathrm{~m}$ away from the rocky wall. Upon returning to the boat, samples were immediately processed as follows: ethanol-wiped forceps were used to remove a collected sample from the conical tube and the sample was dipped multiple times in $0.02 \mu \mathrm{m}$-filtered sea water to remove unattached debris and contaminating overlying sea water. Samples were immediately placed in Ambion RNAlater as per manufacturer's instructions (Thermo Fisher Scientific, Waltham, MA, USA) and stored at $4^{\circ} \mathrm{C}$ for 3 weeks until DNA extraction.

\section{Total DNA Extraction and PCR Amplification of 16S rRNA}

Prior to extraction, samples were processed to remove RNAlater as per the manufacturer instructions. Briefly, each sample was aseptically removed from RNAlater, weighed, and a $50 \mathrm{mg}$ subsample was placed in 450-500 mL $1 \times$ PBS, pH 8.0. Samples were centrifuged for $1 \mathrm{~min}$ at $4000 \times g$, and the supernatant containing residual RNAlater was carefully removed. Pellets were subsequently processed using the PowerPlant Pro DNA Isolation kit (MO BIO Laboratories, CA, USA) according to the manufacturer instructions using a TissueLyser II (Qiagen, Valencia, CA, USA) at $30 \mathrm{~Hz}$ for $10 \mathrm{~min}$. Due to large amounts 
of mucous, the $\mathrm{Sj}$ sample and $50 \mathrm{mg}$ of each $\mathrm{Pl}$ sample were first ground using liquid nitrogen and a sterilized mortar and pestle prior to DNA isolation.

Bacterial and Archaeal V4-V6 regions of the 16S rRNA gene were amplified using primers A519F (CAGCMGCCGCGGTAA; Wang and Qian, 2009) and 1061R (CRRCACGAGCTGACGAC; Andersson et al., 2008) from probeBase (Loy et al., 2007). Final amplification reaction volumes were $25 \mu \mathrm{L}$ and contained $1 \times$ Q5 High-Fidelity $2 \times$ Master Mix (New England Biolabs, Ipswich, MA, USA), $100 \mathrm{ng}$ template, and $1 \mu \mathrm{M}$ of each primer. Reactions were run with a single denaturation step at $98^{\circ} \mathrm{C}$ for $30 \mathrm{~s}$ followed by 30 cycles at $98^{\circ} \mathrm{C}$ for $30 \mathrm{~s}, 59^{\circ} \mathrm{C}$ for $15 \mathrm{~s}$, and $72^{\circ} \mathrm{C}$ for $30 \mathrm{~s}$ and completed with a final extension step of $72^{\circ} \mathrm{C}$ for $2 \mathrm{~min}$. DNA from a previously collected, typical water sample was also amplified using this protocol and running the PCR for 35 cycles. Amplified DNA was visualized on a $1 \%$ agarose gel using SYBR Gold Nucleic Acid Stain, purified using DNA Clean \& Concentrator-5 kits (Zymo Research, Irvine, CA, USA), and eluted with $6 \mu \mathrm{L}$ nuclease-free water (Thermo Fisher Scientific, Waltham, MA, USA). Amplicons were first quantified on a Nanodrop (Thermo Fisher Scientific, Waltham, MA, USA) to ensure the 260/280 absorbance ratio was near 1.8 and then quantified again using the Quant-iT dsDNA HS Assay kit (Invitrogen, Carlsbad, CA, USA) and measured on a Qubit fluorometer (Invitrogen).

\section{Illumina Library Preparation and High-Throughput Sequencing}

Amplicon libraries were prepared for Illumina MiSeq multiplex paired-end sequencing using NEBNext Ultra DNA Library Prep kit and the NEBNext Multiplex Oligos for Illumina Index Primers Sets 1 and 2 (New England Biolabs, Ipswich, MA, USA). Twenty nanograms of amplicon DNA were used for each library preparation reaction. Ampure Beads (Beckman Coulter, Indianapolis, IN, USA) were used for all DNA purification steps following the manufacturer instructions. Adaptor-ligated and indexed samples were visualized for purity and quantification using an Agilent 2100 Bioanalyzer (Agilent, Santa Clara, CA, USA). Twenty samples with final molar concentrations $>1 \mathrm{nM}$ were submitted to the UC Davis Genome Center (Davis, CA, USA) for paired-end, multiplex sequencing on the Illumina MiSeq platform using the MiSeq Reagent Kit v3 (Illumina, San Diego, CA, USA). Cluster generation, sequencing (600 cycles), image processing, demultiplexing, and quality score calculations were all performed on the MiSeq 500 platform (Illumina). Raw read data have been submitted to the NCBI Sequence Read Archive under BioSample Accession numbers SAMN03203155-SAMN03203174 within BioProject Accession number PRJNA268033. We further filtered reads for quality using the IlluminaClip (default settings), and Sliding Window (4 bases, average quality score of $>25$ ) options in Trimmomatic (Bolger et al., 2014).

Sequence assembly and quality control described here were performed using mothur (v 1.32.1-v.1.33.0; Schloss et al., 2009). The MiSeq v3 reagent kit (Illumina) produced read lengths ca. 300 bp. Paired-end reads that included the V4-V6 variable regions and overlapped in the $16 \mathrm{~S}$ ribosomal RNA gene C45 region were processed. Sequence contigs shorter than 501 bp with $>10$ ambiguous bases and homopolymers $>10$ bases were removed. Sequences were aligned to the SILVA SSU Ref Nonredundant (NR) 119 database (Quast et al., 2013) and trimmed to equal alignment length (608 bp with gaps, 552 bp mean sequence length). Chimeric sequences were removed (39,076 sequences or $6.4 \%$, UCHIME (Edgar et al., 2011), and the remaining sequences $(606,679$ unique) were taxonomically classified as described in (Wang and Qian, 2009) using the SILVA SSU Ref NR 119 formatted for mothur.

\section{Sequence Binning and Phylogenetic Analyses}

Sequencing and assembly generated an average of 22,685 assembled 16S rRNA gene fragments per sample (range: 11,063$33,481)$. Sequences were binned into operational taxonomic units (OTUs) of $97 \%$ identity using the Average Neighbor method (Schloss and Westcott, 2011) and resulted in 5594 OTUs. Of these, 3736 were singletons and an additional 70 OTUs were characterized as chloroplasts and were culled from further analyses. Samples were randomly normalized to contain an equal number of sequences for comparative purposes (determined by the sample containing the fewest sequences, Mc_1_12m, 10,448 sequences). Alpha-diversity statistics including Good's Coverage Estimator and the mean number of observed OTUs were then calculated using mothur (v. 1.33.3) and compared using Student's t-test.

Remaining Bray-Curtis Dissimilarity Indices of the abundances of remaining OTUs were calculated to assess betadiversity, and visualized by hierarchical clustering (hclust) using the average neighbor method and nMDS analyses (metaMDS), with $\mathrm{R}$ and the Vegan package (Oksanen et al., 2013). Statistical differences in microbial community composition between different samples (gorgonian vs. non-gorgonian), species (Mc vs. Mf), colonies, and depth (8-9 m vs. 10-12 vs.16 m) were tested for using the adonis function (PERMANOVA test) with 999 permutations, also in Vegan. The OTUs contributing the most to the observed clusters were determined using the Vegan function simper, and verified using the same function on Primer-E (Clarke, 1993). OTU consensus taxonomies were obtained using the SILVA SSU Ref NR 119 database, and OTU sequence representatives were extracted in mothur. OTUs that were unclassifiable beyond Phylum or Class level were examined more closely using the NCBI GenBank NR and 16S ribosomal RNA reference (Bacteria and Archaea) databases (October 2014) and the BLASTN algorithm (Altschul et al., 1990). Reference sequences with the highest percent identity and lowest $e$-values were used to construct a phylogenetic tree (the number of top matches, highest percent identities, and $e$-values varied across OTU representatives). Maximum-likelihood phylogenetic relationships of the OTU representative sequences were assessed using ClustalW (Thompson et al., 2002) to align sequences, and maximum-likelihood trees calculated using the Jukes-Cantor model of substitution (Jukes and Cantor, 1969) with 1000 bootstrap replicates (Geneious v.5.6.4). 
Relative abundances of normalized microbiomes were compared across samples bacterial classes using a Bubble Plot in MS Excel. Pie charts of the same data were composed excluding major OTUs (determined from the SIMPER analyses) for each species of Muricea to examine underlying diversity. The OTU representative sequence data have been submitted to the GenBank database under accession numbers KP174126-KP174134.

\section{Microscopic Observations and Mucus Production}

Endozoic cells were imaged in vivo using a BX51 epifluorescent microscope equipped with a DP70 digital camera (Olympus) using the "chlorophyll" filter set (excitation: $480 \mathrm{~nm}$, emission: $660 \mathrm{~nm})$.

Four colonies of Mc and two colonies of Mf were collected and maintained in tanks with unfiltered, flow through sea water, for four weeks during June 2012. Colonies were exposed to natural light conditions. All colonies were examined for the percentage of the total colony that was visibly covered with mucus. When visible, samples of mucus were collected with a sterile syringe, incubated with SYBR Green nucleic acid stain as per the manufacturer's instructions (Bio-Rad), and examined using an Olympus BX5100 epi-fluorescent microscope.

\section{RESULTS}

Good's coverage estimated a mean of $96.3 \%$ for all organismal samples (range: 92.3-98.3\%), indicating adequate sampling effort (Supplemental Table S1). SW had the lowest coverage (83.4\%) but the greatest number of observed OTUs, while all organismassociated microbiomes exhibited less diversity (Figure 1). Normalized Mf samples consistently had more observed OTUs than $\mathrm{Mc}\left(t_{15}=2.44, p=0.027\right)$.

The microbiomes produced distinct sample-specific clusters whereby taxonomic assemblages from each sample were 20\% similar to the other samples within the cluster (Figure 2). OTUs primarily contributing to the observed clusters in Figure 2 were OTUs 1, 3, 4, and 7 according to the SIMPER analysis.

Of the tested variables, host species type had the greatest effect in determining octocoral microbiome compositions accounting for $58.2 \%$ of the observed variability (PERMANOVA $\left.F_{1,15}=19.5, p=0.001\right)$. Muricea microbiomes all contained an average of $15 \%$ unclassified Bacteria compared to the SW and $\mathrm{Pl}$ samples where $2-10 \%$ of sequences were unclassifiable (Figure 3). Sequences clustered into OTU1 composed 6-64\% of Mc microbiomes, compared to $<0.001 \%$ of any other sampled microbiome, and were noticeably abundant in the Mc_12m and Mc_3_16m microbiomes. Phylogenetic analyses indicated OTU1 was at least 96\% identical to OTU7 and 85\% identical to NR_044756, Spirochaeta halophila (Figure 4). This observation is corroborated by our microscopic examinations of Spirochaetes in Mc mucus (Figure 5). OTU4 represented 6-61\% of Mc microbiome sequences, vs. $<0.3 \%$ in all other samples, while OTU3 composed 12-62\% of Mf microbial communities, compared to $<0.05 \%$ of all other sample microbiomes (Figure 3 ).
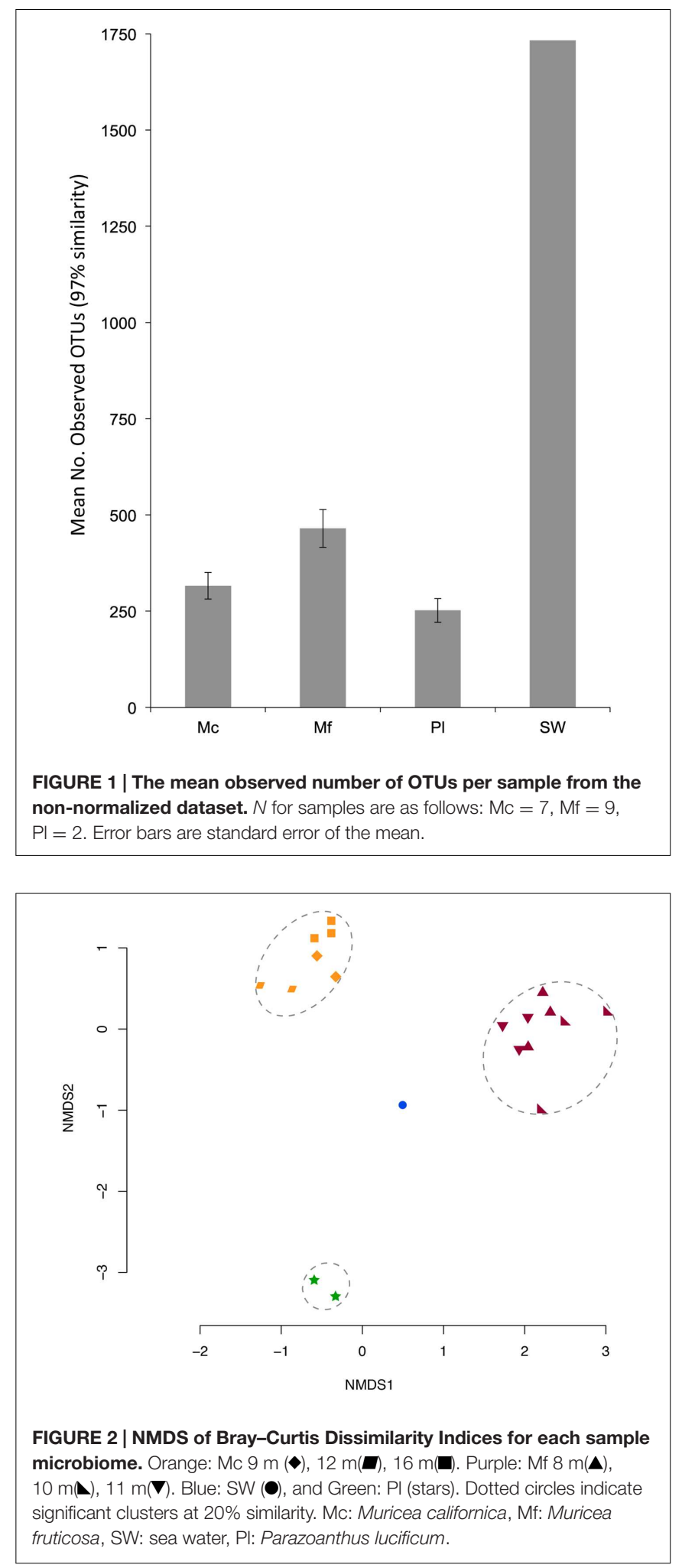

OTUs 3 and 4 were $87.5 \%$ identical to each other, and representative sequences formed a monophyletic group with multiple Mycoplasma sequences isolated from Muricea elongata, a sister gorgonian species found in the coastal Gulf of Mexico and 


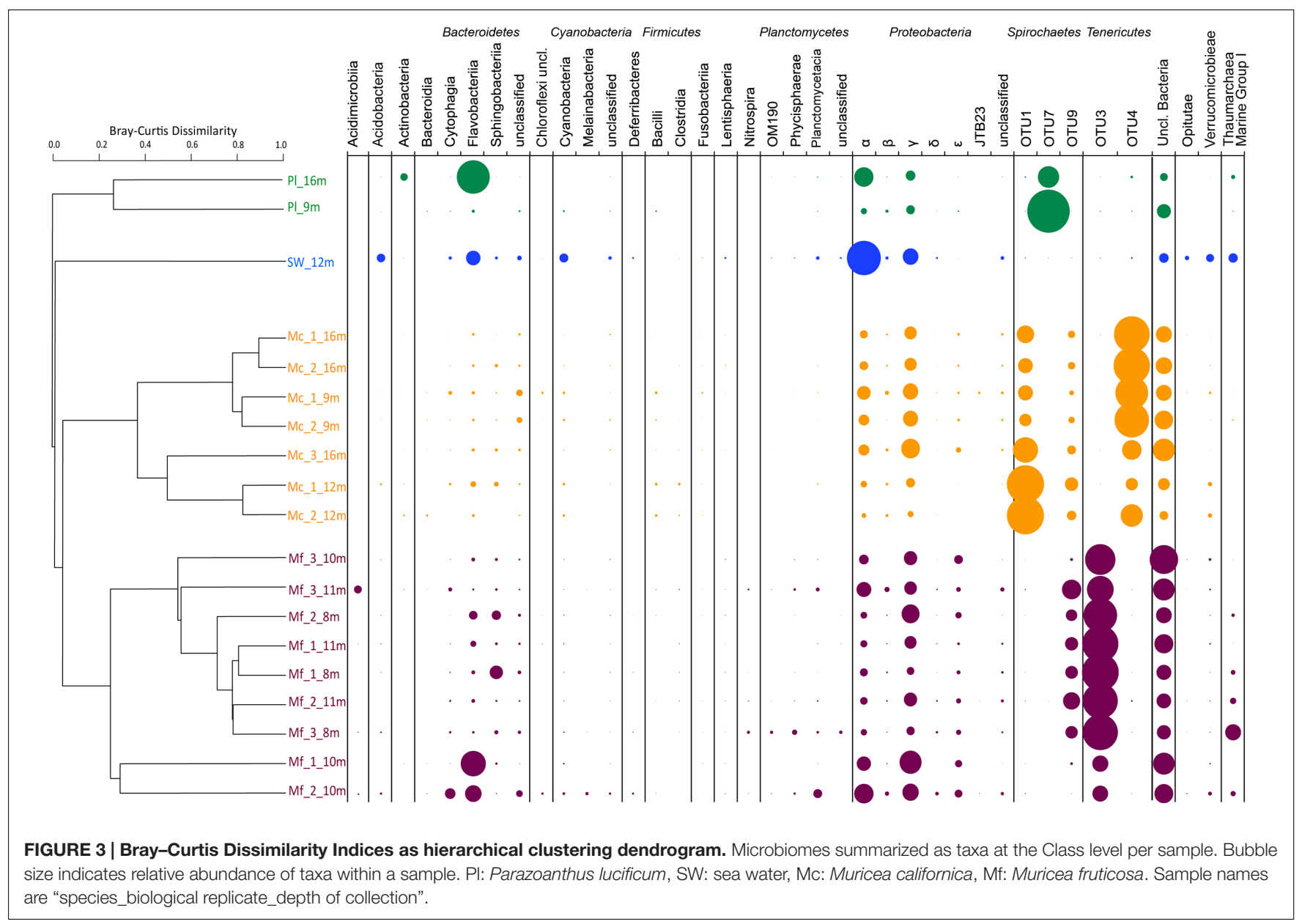

the Caribbean (NCBI PopSet: 134140623, Ranzer et al., 2007). OTU4, found in the Mc samples, formed a monophyletic group with Mycoplasma sequences isolated from healthy M. elongata colonies, while OTU3 grouped with those from bleached, diseased M. elongata colonies (Figure 4).

Overall, Muricea microbiomes had on average more $\gamma$-Proteobacteria sequences than $\alpha$-Proteobacteria $(t=2.03$, $d f=80, p=0.05$; Figure 5), with the exception of Mf_3_11m, and Mf_2_10m, due to a large number of sequences from $\alpha$-Proteobacteria OTU75. Other taxa showing Muricea spp. specificity included $\alpha$-Proteobacteria such as Thalassospira (observed in 5 of $7 \mathrm{Mc}$ samples, and no Mf samples), Nitratireductor (all Mc samples, no Mf sample), and $\gamma$-Proteobacteria like Endozoicomonas, Caedibacter and Francisella (observed in all Muricea samples, but higher abundances in Mc samples; Caedibacter and Francisella observed in 6 of 7 Mc samples; Figure 5). Also, Vibrio spp. $(\gamma$-Proteobacteria) sequences were observed in all Muricea samples, but more so in Mf samples (1-7\% of Mf communities, $<0.4 \%$ of Mc communities). Candidatus nitrosopumilus and Sulfuricurvum were observed in all Mf samples and no Mc samples ( $1-2 \%$ of mean Mf community composition). Overall, Muricea microbiomes had on average more $\gamma$-Proteobacteria sequences than $\alpha$-Proteobacteria $(t=2.03, d f=80, p=0.05$;
Figure 5), with the exception of Mf_3_11m, and Mf_2_10m, due to a large number of sequences from $\alpha$-Proteobacteria OTU75. OTU75 was $98 \%$ identical to uncultured Sinorhizobium, a clonal sequence isolated from bleached $M$. elongata (Figure 4, NCBI PopSet: 134140623, Ranzer et al., 2007).

Variation between Mc colony microbiomes was significant (PERMANOVA: $F_{1,6}=7.45, R^{2}=0.60, p=0.025$ ). Colony Mc_9m was distinct from other Mc colonies because more Francisella ( $\gamma$-Proteobacteria) sequences were observed in each of the replicate branch communities (Figure 3, dendrogram). As previously stated, the high number of OTU1 sequences defined the Mc_12m microbiomes. Depth was not a significant factor in contributing to these observed differences.

Mf colony microbiomes were also significantly different from each other (PERMANOVA: $F_{1,6}=4.33, R^{2}=0.38, p=0.007$ ). Colonies from $8 \mathrm{~m}$ and $11 \mathrm{~m}$ clustered together and separate from the Mf_10m colony. Approximately $80 \%$ of the $8-\mathrm{m}$ and $11-\mathrm{m}$ replicate branch microbiomes were dominated by OTU9 (6-17\% of colony replicate branch microbiomes) and previously described OTU4 (Figure 3). OTU9 was $94 \%$ identical to OTU1, and $>80 \%$ identical to NR_044576, S. halophila. OTU4 contributed to only $37 \%$ of Mf_10m microbiomes; OTU9 was minimally observed. This, and the relatively high abundance of Family NB1d sequences ( $\gamma$-Proteobacteria OTU54, 


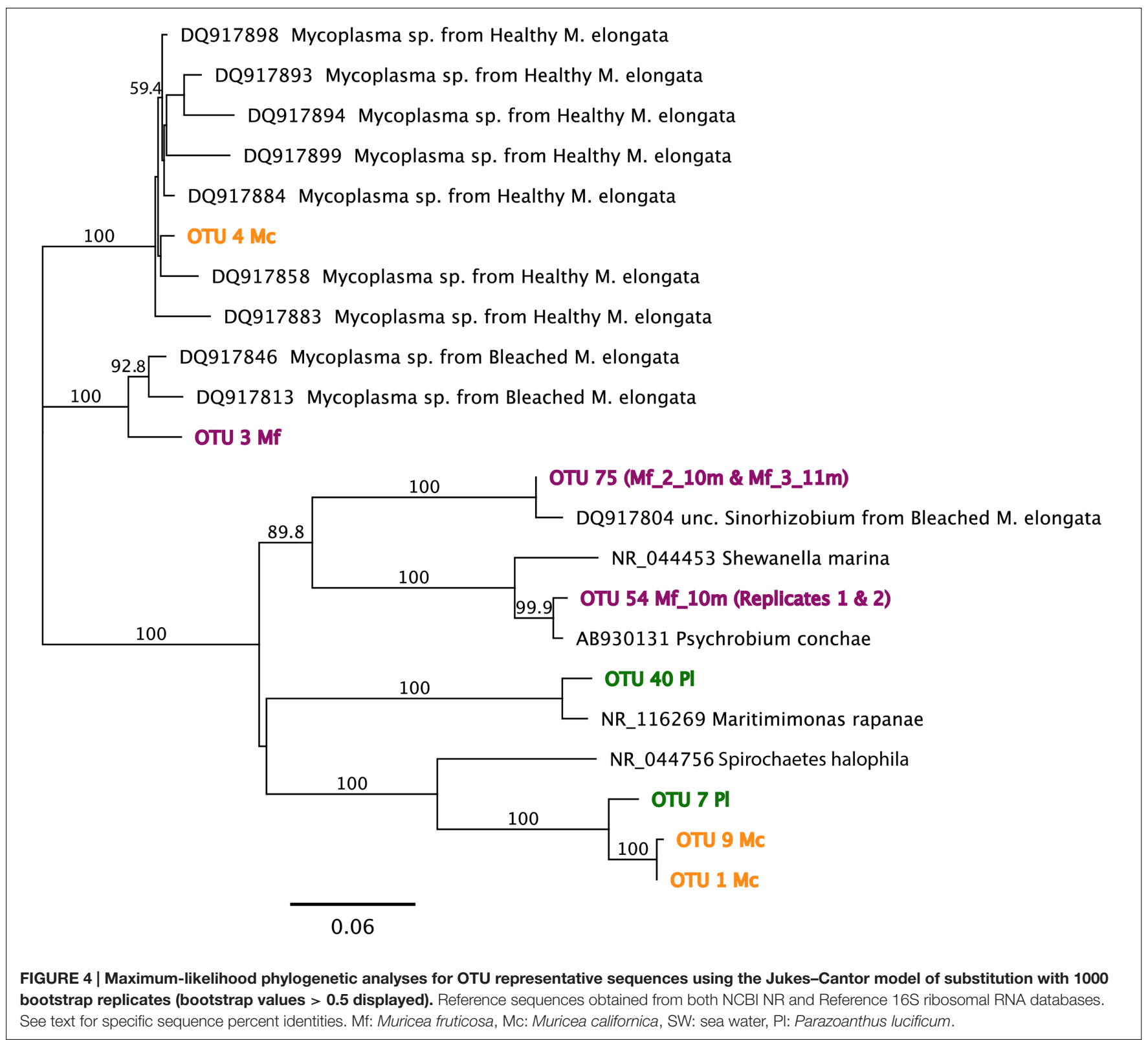

99\% identical to AB930131, Psychrobium conchae, Figure 4), and Tenacibaculum (Flavobacteria) sequences produced the clustering of branch replicate samples 1 and 2 from colony Mf_10m. Colony Mf_8m replicate branch microbiomes were distinct from Mf_11m because of the relatively high abundance of Sphingobacteria Saprospiracea (Bacteroidetes) and the Thaumarchaea C. nitrosopumilus (Marine Group I). Again, depth did contribute significantly in explaining this variation.

The sea water microbiome was different from all organismassociated microbiomes, dominated by $\alpha$-Proteobacteria, namely Candidatus Pelagibacter (53\% of microbiome) and $\gamma$-Proteobacteria SAR86 clade (12\% of microbiome; Figure 3). These specific taxa minimally contributed $(<0.13 \%)$ to all other microbiomes.
Pl microbiomes were also distinct. The relative abundance of OTU7 primarily distinguished the $\mathrm{Pl}$ microbiomes from all other samples (Figure 3; 21\% of Pl_16m, 84\% in Pl_9m, and less than $0.03 \%$ of any other sample communities), and most closely related to NR_044756, Spirochaeta halophila (85\% identical, Figure 4). Pl_16m harbored a large number of Flavobacteriaceae sequences (Figure 3, 48\% of community), specifically OTU40 (47.8\% of community), which was $97 \%$ identical to NR_116269, Maritimimonas rapanae (Figure 4). OTU40 contributed $<0.04 \%$ to any other microbiome, including Pl_9m.

Many features of the octocoral microbiomes were suggestive of photosymbiont-containing invertebrates including the observed strains of Mycoplasma, the presence of Endozoicomonas, and a higher $\gamma$-: $\alpha$-Proteobacteria ratio in Mc samples. Thus, we examined the polyps of both species of 

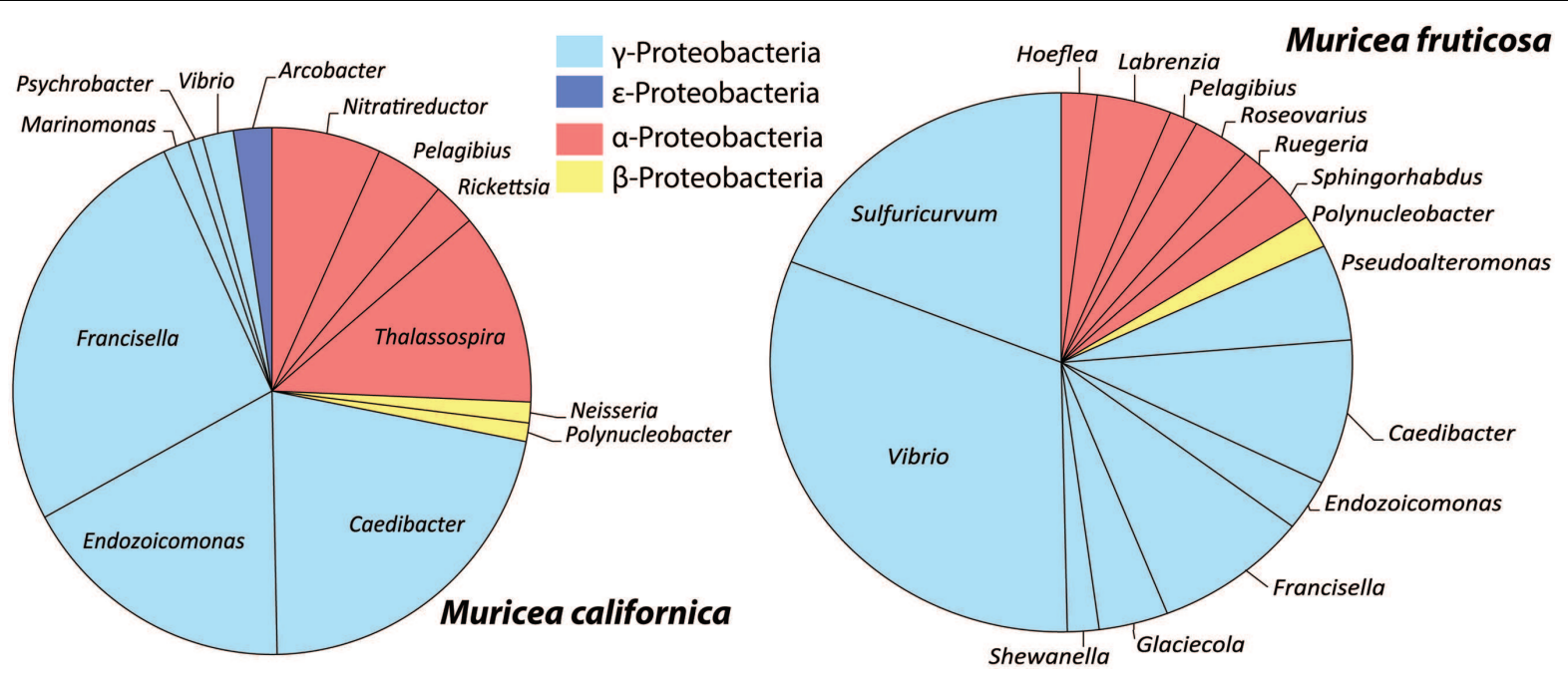

FIGURE 5 | Mean relative abundances of Proteobacteria taxa from M. californica and M. fruticosa.

Muricea using transmitted light and epifluorescence microscopy to determine if photosynthesizing algae were present. Indeed, 5-15 $\mu \mathrm{m}$ brown cells were observed in abundance within $\mathrm{Mc}$ polyps and fluoresced red when excited with blue light, indicating that the cells contain chlorophyll (Figure 6). Pigmented cells were not observed in Mf polyps (data not shown).

Mc colonies produced mucus daily and, in general, mucus production appeared to be greater during daylight hours. Mucus was almost never observed on Mf colonies (Figure 7). Spirillumshaped bacteria were observed in mucus from Mc (Supplemental Figure S1).

\section{DISCUSSION}

The microbiomes of two co-occurring and ecologically important temperate gorgonian octocoral species, M. californica and M. fruticosa, were compared to overlying sea water and nearby zoanthid associated microbiomes. We confirmed that host-associated microbial assemblages exist and are distinct from those in surrounding sea water (Figure 3) and other nearby benthic organisms ( $P$. lucificum). Using inter- and intracolony replication, we also observed that the microbiomes of each species of Muricea each have specific and predictable compositions.

Our results revealed specific relationships between hosts and members of their associated microbial communities. Both species of Muricea had an abundance of sequences from the bacterial phylum, Tenericutes, specifically from the genus, Mycoplasma. This relationship has been observed in deep-sea gorgonians (Gray et al., 2011), the cold water coral, Lophelia pertusa (Kellogg et al., 2009), and a species of gorgonian from the Atlantic Ocean, Muricea elongata (Ranzer et al., 2007). The data of Ranzer et al. (2007) also showed two strains of Mycoplasma associating separately with different colonies of $M$. elongata, but the distinction between those colonies was healthy and bleached.

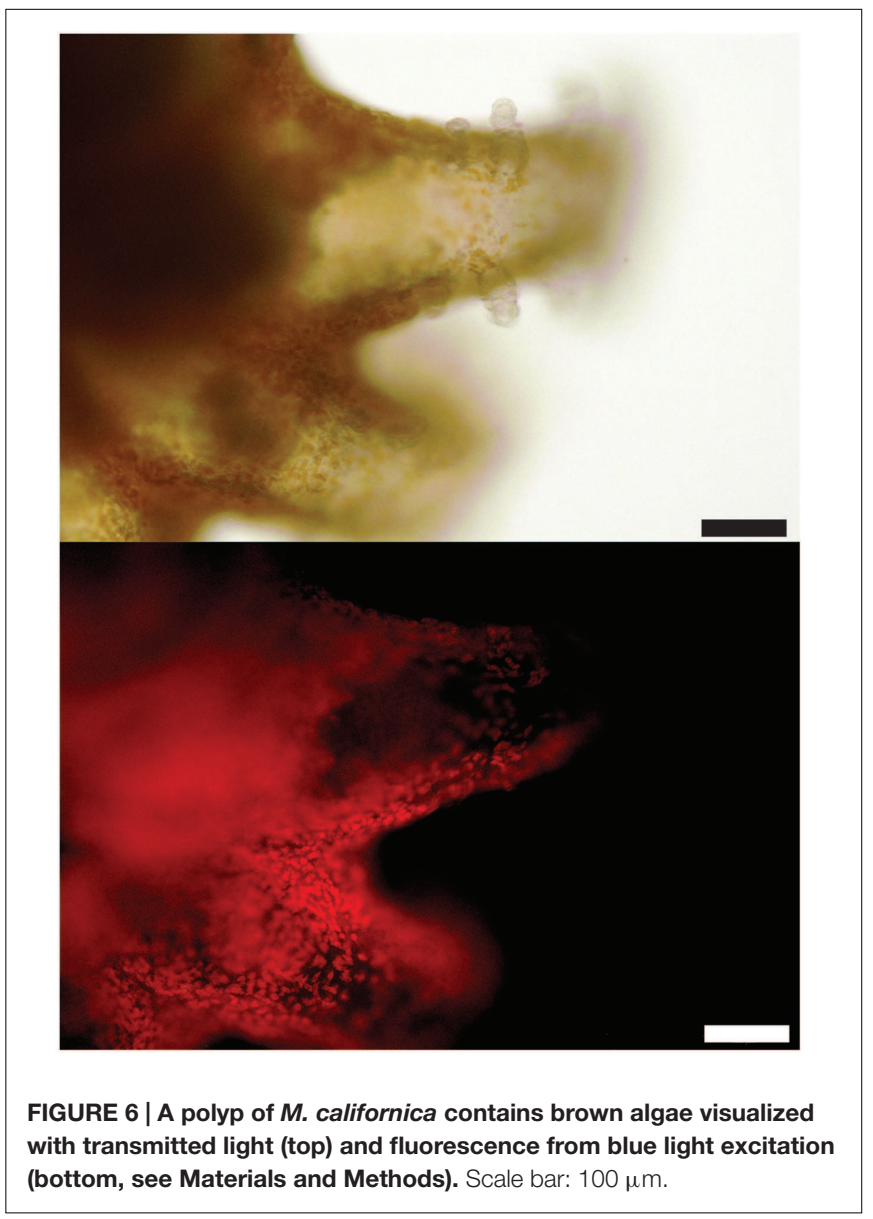

We saw a similar relationship: Mycoplasma strains associated with healthy $M$. elongata were also found in photosymbiontcontaining M. californica microbiomes, and Mycoplasma strains 


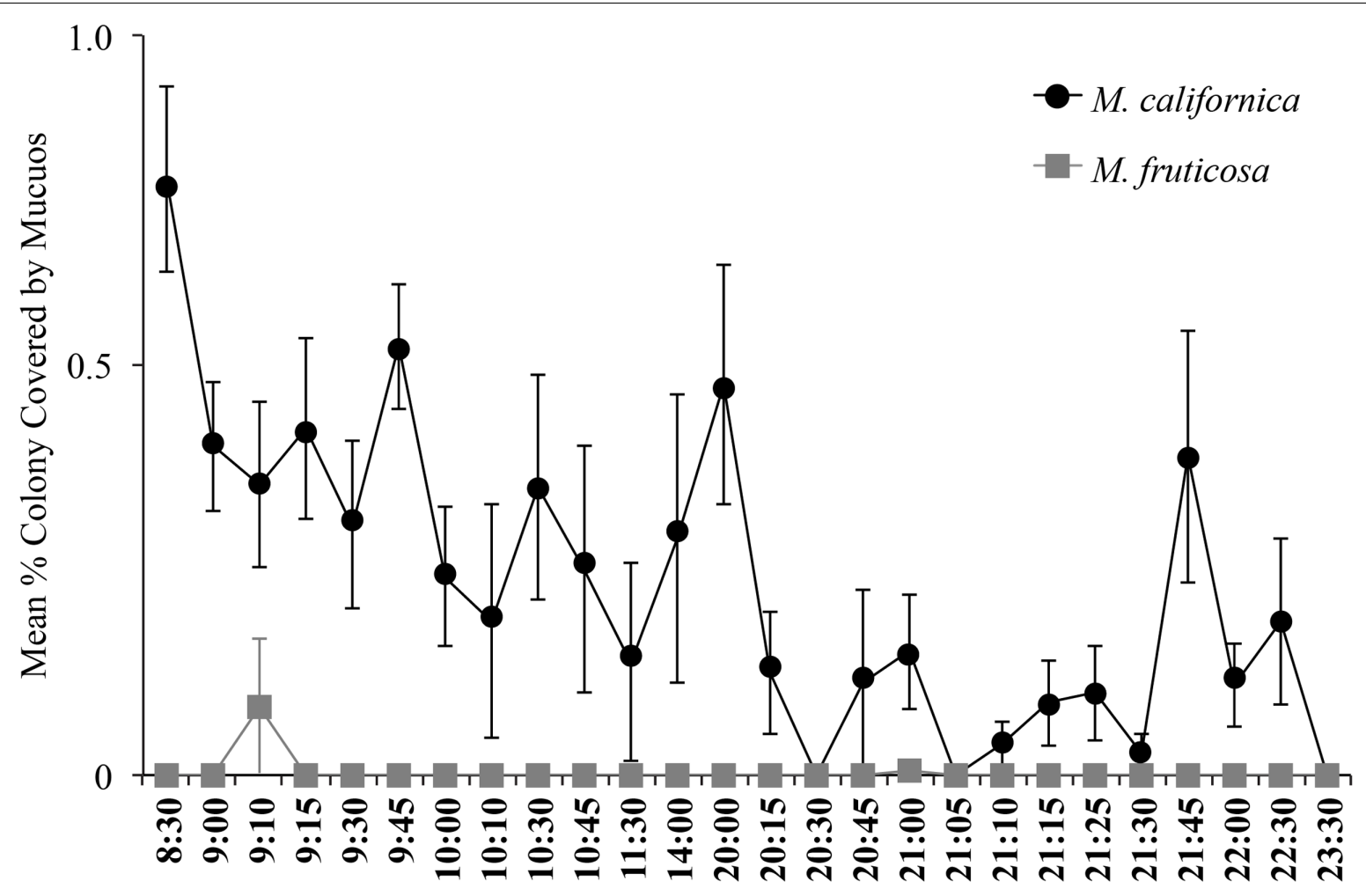

FIGURE 7 | Mucous coverage was almost continuously observed on $M$. californica $(\boldsymbol{n}=\mathbf{4}$ colonies) and little to not at all on Muricea fruticosa ( $\boldsymbol{n}=\mathbf{2}$ colonies). Colonies were observed daily from June 6, 2013 to September 19, 2013. Means were calculated from 2 to 24 total observations, depending on the time point.

associated with bleached $M$. elongata were observed in white polyp $M$. fruticosa microbiomes.

Mycoplasma requires secondary metabolite sterols and fatty acids (FAs) for growth (Ludwig et al., 2010). Such compounds are produced by Muricea (Popov et al., 1983; Gutiérrez et al., 2006), but the types of FA produced are highly dependent on the coral diet: FA produced by and transferred from symbiotic photosynthetic algae to the host coral are compositionally different than those produced by the coral's own biosynthesis pathways (Imbs et al., 2007). This and our observation of chlorophyll-containing cells in the polyps of $M$. californica leads us to hypothesize that different strains of Mycoplasma consistently associate with either $M$. californica or M. fruticosa because of the different FA each produce.

Another major distinction between the microbial communities of co-occurring Muricea species was the greater abundance of sequences from the phylum Spirochaetes in $M$. californica samples. The genera Spirochaeta are chemoheterotrophic and can thrive in a variety of environments (Ludwig et al., 2010). Spirochaetes have been observed in other cnidarian groups including the cold-water coral Lophelia (Kellogg et al., 2009), hydra Hydra attenuata (Hufnagel and Myhal, 1977), and the mucous of sea pens Pennatula phospherea and Pteroides spinosum (Porporato et al., 2013). We observed Spirochaetes-like bacteria in the mucus of $M$. californica (Supplemental Figure S1) and hypothesize that the greater relative abundance of sequences in $M$. californica samples may be due to the daily mucus production and sloughing performed by this species, which was not been observed in M. fruticosa.

Muricea microbiomes consisted of relatively more $\gamma$-Proteobacteria sequences than $\alpha$-Proteobacteria. Recently, Bourne et al. (2013) showed that photosymbiontcontaining marine invertebrates had a greater abundance of $\gamma$-Proteobacteria. Those without photosymbionts generally maintained relatively more $\alpha$-Proteobacteria. The photosymbionts observed in $M$. californica polyps may contribute to the abundance of $\gamma$-Proteobacteria sequences observed. The $\gamma$-Proteobacteria genera from $M$. californica samples were limited to Endozoicomonas, Caedibacter, and Francisella. Endozoicomonas sequences have been isolated from photosymbiont-containing corals and gorgonians (Bayer et al., 2013; Bourne et al., 2013) and metabolize the organic sulfur compound, dimethylsulfoniopropionate (DMSP), a byproduct of photosynthetic algae (Raina et al., 2009). The observed photosymbionts may be producing DMSP and therefore influencing the presence of Endozoicomonas. Interestingly, M. fruticosa had similar $\gamma$-Proteobacteria genera but in lower relative abundances. Vibrio sequences were the most abundant $\gamma$-Proteobacteria sequences found. Members of the genus Vibrio are among the known pathogens of corals (Bally and Garrabou, 2007; Mouchka et al., 2010; Vezzulli et al., 2010), and diseased corals tend to harbor greater microbial diversity 
compared to healthy conspecifics (Bourne et al., 2008; Sunagawa et al., 2009). While M. fruticosa colonies may simply harbor more microbial diversity naturally, the greater OTU diversity and number of Vibrio sequences, in addition to the particular strain of Mycoplasma observed could be indicative of microbial community instability and possibly a diseased state.

The sequences of nitrifying microbes found uniquely amongst the host-associated microbiomes of the Muricea were of particular interest. M. fruticosa samples contained the Archaeon C. nitrosopumilus, a known ammonia oxidizer (Francis et al., 2007), and M. californica had an abundance of Nitratireductor, a nitrate-reducing bacteria (Labbé et al., 2004). Nitrogen cycling in corals has been observed (Shashar et al., 1994), and a model of the role Nitrosopumilus may play in ammonia oxidation has been presented (Siboni et al., 2008). Nitrogenous waste (dissolved inorganic nitrogen, DIN) from coral colonies is either released into the surrounding sea water, in the case of azooxanthellate corals, or transferred to photosymbionts, if present (Dubinsky and Jokiel, 1994). In fact, the density, chlorophyll $a$ content, and rate of photosynthesis of coral photosymbionts is tightly coupled to the availability of DIN (Hoegh-Guldberg and Smith, 1989). Thus, Muricea metabolic waste products may offer a distinct niche for the observed nitrifying microbes, and the presence of the chlorophyll-containing cells may impact this diversity by altering the amounts and types of DIN released.

Interestingly, the gorgonian samples both contained a high number of novel sequences, those that were unclassifiable beyond the domain level, because there were no reference sequences greater than $80 \%$ identical. This proportion of sequences implies that these corals may be important sources of novel biological diversity. Gorgonians have been targeted as promising sources of novel secondary metabolites or marine natural products (MNPs; Rocha et al., 2011). But in most cases, an associated microbe, and not the invertebrate host, is producing the compound(s) of interest (Rath et al., 2011) and our study offers novel information about the microbial organisms associated with gorgonians that may be relevant targets for bio-discovery.

Gorgonian microbiomes, in general, are extremely understudied, especially compared to their scleractinian relatives. While a $16 \mathrm{~S}$ rRNA gene diversity study is not definitive of functional or metabolic interactions in a metaorganism, a comparative approach to microbial diversity, as we have

\section{REFERENCES}

Altschul, S. F., Gish, W., Miller, W., Myers, E. W., and Lipman, D. J. (1990). Basic local alignment search tool. J. Mol. Biol. 215, 403-410. doi: 10.1016/S00222836(05)80360-2

Andersson, A. F., Lindberg, M., Jakobsson, H., Bäckhed, F., Nyrén, P., and Engstrand, L. (2008). Comparative analysis of human gut microbiota by barcoded pyrosequencing. PLoS ONE 3:e2836. doi: 10.1371/journal.pone.0002836

Bally, M., and Garrabou, J. (2007). Thermodependent bacterial pathogens and mass mortalities in temperate benthic communities: a new case of emerging disease linked to climate change. Glob. Change Biol. 13, 2078-2088. doi: 10.1111/j.13652486.2007.01423.x

Bayer, T., Arif, C., Ferrier-Pagès, C., Zoccola, D., Aranda, M., and Voolstra, C. R. (2013). Bacteria of the genus Endozoicomonas dominate the microbiome of the described here, can highlight and substantiate significant differences between the available niches provided by various host organisms.

\section{AUTHOR CONTRIBUTIONS}

$\mathrm{JH}$ and $\mathrm{KH}$ designed the experiment. JH collected, prepared, and analyzed the samples. $\mathrm{JH}$ produced the figures and wrote the manuscript. $\mathrm{KH}$ advised on the figure production as well as the manuscript preparation.

\section{FUNDING}

The USC-Wrigley Rose Hills Summer Graduate Research Fellowship supported this project.

\section{ACKNOWLEDGMENTS}

We are grateful to Kellie Spafford and Lorraine Sadler and Wrigley Marine Science Center Staff for assistance in sample collections. Undergraduate summer fellows, Nicole McNabb and Samantha Wright Leigh, were integral to gorgonian data collection. They thank David Caron, Benjamin Tully, Rohan Sachdeva, Jay Liu, Jacob Cram, Ella Sieradzki, and three anonymous reviewers for their invaluable advice on sequence analysis and manuscript review. Support was provided by a USC Wrigley Institute for Environmental Studies Rose Hills Summer Fellowship. These data contributed to a dissertation thesis (Holm, 2015).

\section{SUPPLEMENTARY MATERIAL}

The Supplementary Material for this article can be found online at: http://journal.frontiersin.org/article/10.3389/fmicb. 2016.00917

FIGURE S1 | Muricea californica mucous contains Spirochaetes-like cells. Mucus 30 from M. californica stained with SYBR Gold and examined using fluorescence microscopy.

Mediterranean gorgonian coral Eunicella cavolini. Mar. Ecol. Prog. Ser. 479, 75-84. doi: 10.3354/meps10197

Bolger, A. M., Lohse, M., and Usadel, B. (2014). Trimmomatic: a flexible trimmer for Illumina sequence data. Bioinformatics 30, 2114-2120. doi: 10.1093/bioinformatics/btu170

Bosch, T. C. G., and McFall-Ngai, M. J. (2011). Metaorganisms as the new frontier. Zoology (Jena) 114, 185-190. doi: 10.1016/j.zool.2011. 04.001

Bourne, D., Iida, Y., Uthicke, S., and Smith-Keune, C. (2008). Changes in coralassociated microbial communities during a bleaching event. ISME J. 2, 350-363. doi: 10.1038/ismej.2007.112

Bourne, D. G., Dennis, P. G., Uthicke, S., Soo, R. M., Tyson, G. W., and Webster, N. (2013). Coral reef invertebrate microbiomes correlate with the presence of photosymbionts. ISME J. 7, 1452-1458. doi: 10.1038/ismej. 2012.172 
Bourne, D. G., and Webster, N. S. (2013). "Coral reef bacterial communities," in The Prokaryotes, eds E. Rosenberg, E. F. DeLong, S. Lory, E. Stackebrandt, and F. Thompson (New York, NY: Springer Science \& Business Media), 163-187. doi: 10.1007/978-3-642-30123-0_48

Brück, T. B., Brück, W. M., Santiago-Vázquez, L. Z., McCarthy, P. J., and Kerr, R. G. (2007). Diversity of the bacterial communities associated with the azooxanthellate deep water octocorals Leptogorgia miniata, Iciligorgia schrammi, and Swiftia exserta. Mar. Biotechnol. 9, 561-576. doi: 10.1007/s10126-007-9009-1

Clarke, K. R. (1993). Non-parametric multivariate analyses of changes in community structure. Austral. Ecol. 18, 117-143. doi: 10.1111/j.14429993.1993.tb00438.x

Correa, H., Haltli, B., Duque, C., and Kerr, R. (2013). Bacterial communities of the gorgonian octocoral Pseudopterogorgia elisabethae. Microb. Ecol. 66, 972-985. doi: 10.1007/s00248-013-0267-3

Cutress, C. E., and Pequegnat, W. E. (1960). Three new species of Zoantharia from California. Pac. Sci. 14, 89-100.

Dubinsky, Z., and Jokiel, P. L. (1994). Ratio of energy and nutrient fluxes regulates symbiosis between zooxanthellae and corals. Mar. Biol. 48, 313-324. doi: 10.1007/bf00349778

Edgar, R. C., Haas, B. J., Clemente, J. C., Quince, C., and Knight, R. (2011). UCHIME improves sensitivity and speed of chimera detection. Bioinformatics 27, 2194-2200. doi: 10.1093/bioinformatics/btr381

Francis, C. A., Beman, J. M., and Kuypers, M. M. M. (2007). New processes and players in the nitrogen cycle: the microbial ecology of anaerobic and archaeal ammonia oxidation. ISME J. 1, 19-27. doi: 10.1038/ismej.2007.8

Gray, M. A., Stone, R. P., McLaughlin, M. R., and Kellogg, C. A. (2011). Microbial consortia of gorgonian corals from the Aleutian Islands. FEMS Microbiol. Ecol. 76, 109-120. doi: 10.1111/j.1574-6941.2010.01033.x

Grigg, R. W. (1970). Ecology and Population Dynamics of the Gorgonians, Muricea californica and Muricea fruticosa. Coelentera: Anthozoa. Ph.D. thesis, University of California San Diego, 1-280.

Gutiérrez, M., Capson, T. L., Guzmán, H. M., González, J., Ortega-Barría, E., and Quiñoá, E. (2006). Antiplasmodial metabolites isolated from the marine octocoral Muricea austera. J. Nat. Prod. 69, 1379-1383. doi: 10.1021/np060007f

Hoegh-Guldberg, O., and Smith, G. J. (1989). Influence of the population density of zooxanthellae and supply of ammonium on the biomass and metabolic characteristics of the reef corals Seriatopora hystrix and Stylophora pistillata. Mar. Ecol. Prog. Ser. 57, 173-186. doi: 10.3354/meps057173

Holm, J. B. (2015). The Microbiome of Gorgonian Octocorals, Muricea, with a Description of a Novel, Photosynthetic Protistan Symbiont. Ph.D. thesis, University of Southern California, 1-72.

Hufnagel, L. A., and Myhal, M. L. (1977). Observations on a Spirochaete symbiotic in Hydra. Trans. Am. Microsc. Soc. 96, 406-411. doi: 10.2307/ 3225874

Imbs, A. B., Latyshev, N. A., Zhukova, N. V., and Dautova, T. N. (2007). Comparison of fatty acid compositions of azooxanthellate Dendronephthya and zooxanthellate soft coral species. Comp. Biochem. Physiol. B Biochem. Mol. Biol. 148, 314-321. doi: 10.1016/j.cbpb.2007.06.009

Jukes, T., and Cantor, C. (1969). "Evolution of protein molecules," in Mammalian Protein Metabolism, ed. H. N. Munro (New York, NY: Academic Press), 21-132. doi: 10.1016/B978-1-4832-3211-9.50009-7

Kellogg, C. A., Lisle, J. T., and Galkiewicz, J. P. (2009). Culture-independent characterization of bacterial communities associated with the cold-water coral Lophelia pertusa in the northeastern Gulf of Mexico. Appl. Environ. Microbiol. 75, 2294-2303. doi: 10.1128/AEM.02357-08

La Rivière, M., Roumagnac, M., Garrabou, J., and Bally, M. (2013). Transient shifts in bacterial communities associated with the temperate gorgonian Paramuricea clavata in the Northwestern Mediterranean Sea. PLOS ONE 8:e57385. doi: 10.1371/journal.pone.0057385

Labbé, N., Parent, S., and Villemur, R. (2004). Nitratireductor aquibiodomus gen. nov., sp. nov., a novel alpha-proteobacterium from the marine denitrification system of the Montreal Biodome (Canada). Int. J. Syst. Evol. Microbiol. 54, 269-273. doi: 10.1099/ijs.0.02793-0

Loy, A., Maixner, F., Wagner, M., and Horn, M. (2007). probeBase-an online resource for rRNA-targeted oligonucleotide probes: new features. Nucleic Acids Res. 35, D800-D804. doi: 10.1093/nar/gkl856
Ludwig, W., Euzéby, J., and Whitman, W. B. (2010). "Volume 4: The Bacteroidetes, Spirochaetes, Tenericutes (Mollicutes), Acidobacteria, Fibrobacteres, Fusobacteria, Dictyoglomi, Gemmatimonadetes, Lentisphaerae, Verrucomicrobia, Chlamydiae, and Planctomycetes," in Bergey's Manual of Systematic Bacteriology, eds N. R. Krieg, W. Ludwig, W. B. Whitman, B. P. Hedlund, B. J. Paster, J. T. Staley, et al. (New York, NY: Springer), 949. doi: 10.1007/978-0-387-68572-4_1

Mouchka, M. E., Hewson, I., and Drew Harvell, C. (2010). Coral-associated bacterial assemblages: current knowledge and the potential for climate-driven impacts. Integr. Comp. Biol. 50, 662-674. doi: 10.1093/icb/icq061

Oksanen, J., Blanchet, F. G., Kindt, R., Legendre, P., Minchin, P. R., OHara, R. B., et al. (2013). Community Ecology Package. Available at: http://vegan.r-forge.rproject.org/ [accessed September 10, 2014]

Popov, S., Carlson, R. M., and Djerassi, C. (1983). Occurrence and seasonal variation of 19-norcholest-4-en-3-one and 3 beta-monohydroxy sterols in the Californian gorgonian, Muricea californica. Steroids 41, 537-548. doi: 10.1016/0039-128X(83)90093-4

Porporato, E. M. D., Giudice, A., Michaud, L., Domenico, E., and Spanò, N. (2013). Diversity and antibacterial activity of the bacterial communities associated with two Mediterranean sea pens, Pennatula phosphorea and Pteroeides spinosum (Anthozoa: Octocorallia). Microb. Ecol. 66, 701-714. doi: 10.1007/s00248-0130260-x

Quast, C., Pruesse, E., Yilmaz, P., Gerken, J., Schweer, T., Yarza, P., et al. (2013). The SILVA ribosomal RNA gene database project: improved data processing and web-based tools. Nucleic Acids Res. 41, D590-D596. doi: 10.1093/nar/gks1219

Raina, J.-B., Tapiolas, D., Willis, B. L., and Bourne, D. G. (2009). Coral-associated bacteria and their role in the biogeochemical cycling of sulfur. Appl. Environ. Microbiol. 75, 3492-3501. doi: 10.1128/AEM.02567-08

Ranzer, L. K., Restrepo, P. F., and Kerr, R. G. (2007). Data from: Microbial Community Profiles of Bleached and WildType Muricea elongata. NCBI PopSet 134140623. Available at: http://www.ncbi.nlm.nih.gov/popset/134140623?report = genbank

Rath, C. M., Janto, B., Earl, J., Ahmed, A., Hu, F. Z., Hiller, L., et al. (2011). Metaomic characterization of the marine invertebrate microbial consortium that produces the chemotherapeutic natural product ET-743. ACS Chem. Biol. 6, 1244-1256. doi: 10.1021/cb200244t

Reshef, L., Koren, O., Loya, Y., Zilber-Rosenberg, I., and Rosenberg, E. (2006). The coral probiotic hypothesis. Environ. Microbiol. 8, 2068-2073. doi: 10.1111/j.1462-2920.2006.01148.x

Rocha, J., Peixe, L., Gomes, N. C. M., and Calado, R. (2011). Cnidarians as a source of new marine bioactive compounds-An overview of the last decade and future steps for bioprospecting. Mar. Drugs 9, 1860-1886. doi: 10.3390/md9101860

Rohwer, F., Seguritan, V., Azam, F., and Knowlton, N. (2002). Diversity and distribution of coral-associated bacteria. Mar. Ecol. Prog. Ser. 243, 1-10. doi: $10.3354 /$ meps 243001

Rosenberg, E., Koren, O., Reshef, L., Efrony, R., and Zilber-Rosenberg, I. (2007). The role of microorganisms in coral health, disease and evolution. Nat. Rev. Microbiol. 5, 355-362. doi: 10.1038/nrmicrol635

Schloss, P. D., and Westcott, S. L. (2011). Assessing and improving methods used in operational taxonomic unit-based approaches for 16S rRNA gene sequence analysis. Appl. Environ. Microbiol. 77, 3219-3226. doi: 10.1128/AEM.02810-10

Schloss, P. D., Westcott, S. L., Ryabin, T., Hall, J. R., Hartmann, M., Hollister, E. B., et al. (2009). Introducing mothur: open-source, platformindependent, community-supported software for describing and comparing microbial communities. Appl. Environ. Microbiol. 75, 7537-7541. doi: 10.1128/AEM.01541-09

Shashar, N., Cohen, Y., Loya, Y., and Sar, N. (1994). Nitrogen-fixation (acetylenereduction) in stony corals - evidence for coral-bacteria interactions. Mar. Ecol. Prog. Ser. 111, 259-264. doi: 10.3354/meps111259

Siboni, N., Ben Dov, E., Sivan, A., and Kushmaro, A. (2008). Global distribution and diversity of coral-associated Archaea and their possible role in the coral holobiont nitrogen cycle. Environ. Microbiol. 10, 2979-2990. doi: 10.1111/j.1462-2920.2008.01718.x

Sinniger, F., Ocaña, O. V., and Baco, A. R. (2013). Diversity of zoanthids (Anthozoa: Hexacorallia) on Hawaiian seamounts: description of the Hawaiian gold coral and additional zoanthids. PLOS ONE 8:e52607. doi: 10.1371/journal.pone.0052607 
Sun, W., Zhang, F., He, L., and Li, Z. (2014). Pyrosequencing reveals diverse microbial community associated with the Zoanthid Palythoa australiae from the South China Sea. Microb. Ecol. 67, 942-950. doi: 10.1007/s00248-0140395-4

Sunagawa, S., DeSantis, T. Z., Piceno, Y. M., Brodie, E. L., DeSalvo, M. K., Voolstra, C. R., et al. (2009). Bacterial diversity and white plague disease-associated community changes in the Caribbean coral Montastraea faveolata. ISME J. 3, 512-521. doi: 10.1038/ismej.2008.131

Thomas, T., Rusch, D., DeMaere, M. Z., Yung, P. Y., Lewis, M., Halpern, A., et al. (2010). Functional genomic signatures of sponge bacteria reveal unique and shared features of symbiosis. ISME J. 4, 1-11. doi: 10.1038/ismej.2010.74

Thompson, J. D., Gibson, T. J., and Higgins, D. G. (2002). Multiple Sequence Alignment Using ClustalW and ClustalX. Hoboken, NJ: John Wiley \& Sons, Inc. doi: 10.1002/0471250953.bi0203s00

Van Oppen, M., Mieog, J., Sánchez, C. A., and Fabricius, K. E. (2005). Diversity of algal endosymbionts (zooxanthellae) in octocorals: the roles of geography and host relationships. Mol. Ecol. 14, 2403-2417. doi: 10.1111/j.1365294X.2005.02545.x

Vega Thurber, R., Willner-Hall, D., Rodriguez-Mueller, B., Desnues, C., Edwards, R. A., Angly, F., et al. (2009). Metagenomic analysis of stressed coral holobionts. Environ. Microbiol. 11, 2148-2163. doi: 10.1111/j.1462-2920.2009.01935.x

Vezzulli, L., Pezzati, E., Huete-Stauffer, C., and Pruzzo, C. (2013). 16srDNA pyrosequencing of the Mediterranean gorgonian Paramuricea clavata reveals a link among alterations in bacterial holobiont members, anthropogenic influence and disease outbreaks. PLoS ONE 8:e66745. doi 10.1371/journal.pone.0067745

Vezzulli, L., Previati, M., Pruzzo, C., Marchese, A., Bourne, D. G., Cerrano, C., et al. (2010). Vibrio infections triggering mass mortality events in a warming Mediterranean Sea. Environ. Microbiol. 12, 2007-2019. doi: 10.1111/j.14622920.2010.02209.x

Wang, Y., and Qian, P.-Y. (2009). Conservative fragments in bacterial 16S rRNA genes and primer design for $16 \mathrm{~S}$ ribosomal DNA amplicons in metagenomic studies. PLOS ONE 4:e7401. doi: 10.1371/journal.pone. 0007401

Webster, N. S., and Bourne, D. (2007). Bacterial community structure associated with the Antarctic soft coral, Alcyonium antarcticum. FEMS Microbiol. Ecol. 59, 81-94. doi: 10.1111/j.1574-6941.2006.00195.x

Conflict of Interest Statement: The authors declare that the research was conducted in the absence of any commercial or financial relationships that could be construed as a potential conflict of interest.

Copyright (c) 2016 Holm and Heidelberg. This is an open-access article distributed under the terms of the Creative Commons Attribution License (CC BY). The use, distribution or reproduction in other forums is permitted, provided the original author(s) or licensor are credited and that the original publication in this journal is cited, in accordance with accepted academic practice. No use, distribution or reproduction is permitted which does not comply with these terms. 